\title{
Derivation of von Weizsäcker Equation Based on Green-Gauss Theorem
}

\author{
Z. ROMANOWSKI \\ Interdisciplinary Centre for Materials Modelling \\ Pawińskiego 5a, 02-106 Warsaw, Poland \\ AND S. KRUKOWSKI \\ Institute of High Pressure Physics of the Polish Academy of Sciences \\ Sokołowska 29/37, 01-142 Warsaw, Poland
}

(Received August 19, 2008; revised version October 19, 2008)

\begin{abstract}
A simple and short derivation of von Weizsäcker equation for kinetic energy functional is presented. The derivation is based on the Green-Gauss theorem and is valid for one-electron systems. In the proof the asymptotic behavior of wave function for the finite systems was used. Two results important for kinetic energy functional evaluation are also derived as consequences of the Green-Gauss theorem.
\end{abstract}

PACS numbers: 31.15.E-, 71.15.Mb, 71.15.Dx

\section{Introduction}

The density functional theory (DFT) $[1-3]$ is a powerful tool to study the molecular systems. First main theorem of this theory states that the ground state of the system can be fully described by electron density $\varrho(\boldsymbol{r}): \mathbb{R}^{3} \rightarrow \mathbb{R}$. Second theorem states that the total energy of the system can be obtained by minimization of the functional $E[\varrho]$ subject to the constraint $\int_{\mathbb{R}^{3}} \varrho(\boldsymbol{r}) \mathrm{d} \boldsymbol{r}=N$, where $N$ is total number of electrons in the system. The DFT theory is the powerful tool, however it has one drawback: the exact kinetic energy functional and exact exchange-correlation functional are unknown and hence the total energy functional is approximated only.

The research in construction of the exchange-correlation functionals has been successful and many precise (although approximate) functionals based on local (LDA) and gradient (GGA) approximation have been proposed [4]. However, the progress in construction of the kinetic energy functionals is not so fruitful. The review of the orbital-free kinetic energy density functionals (OF-KEDF) is presented by Wang [5]. The availability of accurate orbital-free kinetic energy density functionals could avoid the solution of the Kohn-Sham eigenproblem. It has not been constructed and, therefore, the algorithm solving Kohn-Sham eigenproblem based on the linear combination of atomic orbital (LCAO) leads to algebraic generalized eigenvalue problems with solving cost scaling as $\mathcal{O}\left(n^{3}\right)$.

In the classical approach based on the Kohn-Sham eigenproblem one must store the set of eigenfunctions $\left\{\Psi_{i}(\boldsymbol{r}): \mathbb{R}^{3} \rightarrow \mathbb{R}\right\}_{i=1}^{N}$. If the eigenfunction $\Psi_{i}(\boldsymbol{r})$ is represented by $M$ basis functions, then $M N$ coefficients must be evaluated holding the orthogonality constraints $\int_{\mathbb{R}} \Psi_{i}(\boldsymbol{r}) \Psi_{j}(\boldsymbol{r}) \mathrm{d} \boldsymbol{r}=\delta_{i, j}$. The availability of OF-KEDF could reduce the dimension of the problem to $\mathbb{R}^{3}$. More- over, if electron density $\varrho(\boldsymbol{r})$ was represented by $M$ atomic orbitals, only $M$ coefficients could be evaluated without the orthogonality constraint. Further, one could avoid solving eigenvalue problem and solve the minimization problem with constraints only, where the efficient algorithms are available [6].

There are two classical results proved by ThomasFermi and von Weizsäcker [5, 3] concerning OF-KEDF. Based on these models more sophisticated results were presented in Refs. [7-9]. The key point is that ThomasFermi model is exact only for the free electron gas. The Thomas-Fermi model applied to molecular systems gives quite poor results $[10,11]$. More reliable model was introduced by von Weizsäcker, which is derived using the density matrix formalism. For instance von Weizsäcker model is exact for one-electron systems.

In this paper we present the derivation of von Weizsäcker relation for one-electron systems. The derivation is based on the definition of kinetic energy. Additionally, we prove that the kinetic energy functional for many-electron system cannot include $\int_{\mathbb{R}^{3}} \nabla^{2} \varrho(\boldsymbol{r}) \mathrm{d} \boldsymbol{r}$. The derivation is based on the Green-Gauss theorem.

\section{Consequences of the Green-Gauss theorem}

There are many formulations of the Green-Gauss theorem [12], one of them is the following. Let $f, g: \Omega \subset$ $\mathbb{R}^{3} \rightarrow \mathbb{R}$ be two integrable functions and $\Sigma$ be the surface (boundary) of the closed volume $\Omega$. Then it holds

$$
\begin{aligned}
& \int_{\Omega} f(\boldsymbol{r}) \nabla^{2} g(\boldsymbol{r}) \mathrm{d} \boldsymbol{r}=-\int_{\Omega} \nabla f(\boldsymbol{r}) \nabla g(\boldsymbol{r}) \mathrm{d} \boldsymbol{r} \\
& \quad+\oint_{\Sigma} f(\boldsymbol{r}) \nabla g(\boldsymbol{r}) \cdot \boldsymbol{n} \mathrm{d} \sigma,
\end{aligned}
$$

where $\boldsymbol{n}$ is a normal unit vector to the surface $\Sigma$, directed outwards and $\oint$ denotes the surface integral. For 
the special case $f(\boldsymbol{r}) \equiv 1$ this theorem reduces to

$$
\int_{\Omega} \nabla^{2} g(\boldsymbol{r}) \mathrm{d} \boldsymbol{r}=\oint_{\Sigma} \nabla g(\boldsymbol{r}) \cdot \boldsymbol{n} \mathrm{d} \sigma .
$$

Let assume that $g(\boldsymbol{r}) \equiv \varrho(\boldsymbol{r})$ is the electron density of many-electron finite system, hence the integral $\int_{\mathbb{R}^{3}} \varrho(\boldsymbol{r}) \mathrm{d} \boldsymbol{r}$ must be finite and equal to the number of electrons in the system.

Let us denote by $\|\boldsymbol{v}\|$ the length of vector $\boldsymbol{v} \in \mathbb{R}^{3}$. It is well known $[3,13]$ that for finite systems and for large $r=\|\boldsymbol{r}\|$ the wave function $\Psi(\boldsymbol{r})$ decreases exponentially, i.e. $\Psi(\boldsymbol{r})=C \mathrm{e}^{-\alpha r}$, where $C, \alpha>0$. Hence, the electron density behaves as $\varrho(\boldsymbol{r})=\Psi^{2}(\boldsymbol{r})=C^{2} \mathrm{e}^{-2 \alpha r}$. If $\Omega$ is a ball of radius $r$, then the surface $\Sigma$ is a sphere of radius $r$. For this case the integral on the right hand side of Eq. (2) can be easily obtained

$$
I(r)=\oint_{\Sigma} \nabla g(\boldsymbol{r}) \cdot \boldsymbol{n} \mathrm{d} \sigma=-8 \pi \alpha C^{2} r^{2} \mathrm{e}^{-2 \alpha r} .
$$

The limit of this integral is

$$
\lim _{r \rightarrow \infty} I(r)=-8 \pi \alpha C^{2} \lim _{r \rightarrow \infty} r^{2} \mathrm{e}^{-2 \alpha r}=0 .
$$

Moreover, if the radius of the ball $\Omega$ is increased, then $\Omega \rightarrow \mathbb{R}^{3}$. Hence, based on the above relation, we obtain

$$
\int_{\mathbb{R}^{3}} \nabla^{2} \varrho(\boldsymbol{r}) \mathrm{d} \boldsymbol{r}=0 .
$$

It means that the kinetic energy functional $T$ for many-electron system does not need to account explicitly the term proportional to $\int_{\mathbb{R}^{3}} \nabla^{2} \varrho(\boldsymbol{r}) \mathrm{d} \boldsymbol{r}$. As a consequence, in the gradient expansion of the kinetic energy functional

$$
T \approx T_{0}[\varrho]+T_{1}[\nabla \varrho]+T_{2}\left[\nabla^{2} \varrho\right]+\ldots
$$

the terms proportional to $T_{2}\left[\nabla^{2} \varrho\right]$ can be omitted.

\section{One-electron system}

Let us denote by $\Psi(\boldsymbol{r}): \mathbb{R}^{3} \rightarrow \mathbb{R}$ the real wave function of one-electron system. Then, the kinetic energy of this system is given by

$$
T=-\frac{1}{2} \int_{\mathbb{R}^{3}} \Psi(\boldsymbol{r}) \nabla^{2} \Psi(\boldsymbol{r}) \mathrm{d} \boldsymbol{r},
$$

and the electron density is defined as

$$
\varrho(\boldsymbol{r})=\Psi^{2}(\boldsymbol{r}) \text {. }
$$

Applying the $\nabla^{2}$ operator to both sides of the above relation one obtains

$$
\begin{aligned}
& \nabla^{2} \varrho(\boldsymbol{r}) \equiv \nabla^{2} \Psi^{2}(\boldsymbol{r})=2\|\nabla \Psi(\boldsymbol{r})\|^{2} \\
& \quad+2 \Psi(\boldsymbol{r}) \nabla^{2} \Psi(\boldsymbol{r}),
\end{aligned}
$$

where $\|\nabla \Psi(\boldsymbol{r})\|$ denotes length of vector $\nabla \Psi(\boldsymbol{r})$. Integrating the above equation over $\mathbb{R}^{3}$ and substituting Eq. (7) we obtain

$$
\int_{\mathbb{R}^{3}} \nabla^{2} \varrho(\boldsymbol{r}) \mathrm{d} \boldsymbol{r}=2 \int_{\mathbb{R}^{3}}\|\nabla \Psi(\boldsymbol{r})\|^{2} \mathrm{~d} \boldsymbol{r}-4 T,
$$

hence

$$
T=\frac{1}{2} \int_{\mathbb{R}^{3}}\|\nabla \Psi(\boldsymbol{r})\|^{2} \mathrm{~d} \boldsymbol{r}-\frac{1}{4} \int_{\mathbb{R}^{3}} \nabla^{2} \varrho(\boldsymbol{r}) \mathrm{d} \boldsymbol{r} .
$$

Let us apply $\nabla$ operator to both sides of Eq. (8)

$$
\nabla \varrho(\boldsymbol{r}) \equiv \nabla \Psi^{2}(\boldsymbol{r})=2 \Psi(\boldsymbol{r}) \nabla \Psi(\boldsymbol{r}) .
$$

Taking square of both sides in the above equation and substituting Eq. (8) we obtain

$$
\begin{aligned}
& \|\nabla \varrho(\boldsymbol{r})\|^{2}=4 \Psi^{2}(\boldsymbol{r})\|\nabla \Psi(\boldsymbol{r})\|^{2} \\
& \quad=4 \varrho(\boldsymbol{r})\|\nabla \Psi(\boldsymbol{r})\|^{2} .
\end{aligned}
$$

Inverting this relation we get

$$
\|\nabla \Psi(\boldsymbol{r})\|^{2}=\frac{\|\nabla \varrho(\boldsymbol{r})\|^{2}}{4 \varrho(\boldsymbol{r})} .
$$

Finally, substituting Eq. (14) into Eq. (11) we get the kinetic energy functional

$$
T=\frac{1}{8} \int_{\mathbb{R}^{3}} \frac{\|\nabla \varrho(\boldsymbol{r})\|^{2}}{\varrho(\boldsymbol{r})} \mathrm{d} \boldsymbol{r}-\frac{1}{4} \int_{\mathbb{R}^{3}} \nabla^{2} \varrho(\boldsymbol{r}) \mathrm{d} \boldsymbol{r} .
$$

This is an exact kinetic energy functional for one-electron system where the electron density is defined by function $\varrho(\boldsymbol{r}): \mathbb{R}^{3} \rightarrow \mathbb{R}$. It follows from Eq. (15) that kinetic energy functional depends on electron density $\varrho(\boldsymbol{r})$, length of gradient $\|\nabla \varrho(\boldsymbol{r})\|$ and laplacian $\nabla^{2} \varrho(\boldsymbol{r})$. From Sect. 2 it follows that the second term is equal to zero. Dropping the second term in Eq. (15), the von Weizsäcker equation is derived

$$
T=\frac{1}{8} \int_{\mathbb{R}^{3}} \frac{\|\nabla \varrho(\boldsymbol{r})\|^{2}}{\varrho(\boldsymbol{r})} \mathrm{d} \boldsymbol{r} .
$$

¿From the above discussion it follows that von Weizsäcker equation is valid for any one-electron system.

\section{Kinetic energy in DFT}

In this section we provide the derivation of kinetic energy formula commonly applied in DFT. The derivation is based on equations presented in the previous sections. In DFT the kinetic energy of system containing $N$ electrons is given by

$$
T=-\frac{1}{2} \sum_{i=1}^{N} \int_{\mathbb{R}^{3}} \Psi_{i}(\boldsymbol{r}) \nabla^{2} \Psi_{i}(\boldsymbol{r}) \mathrm{d} \boldsymbol{r},
$$

where $\Psi_{i}(\boldsymbol{r}): \mathbb{R}^{3} \rightarrow \mathbb{R}$ for $i=1, \ldots, N$ is set of occupied one-electron Kohn-Sham eigenfunctions [2, 1, 11, 10, 3]. If each eigenfunction $\Psi_{i}(\boldsymbol{r})$ is occupied by one electron, then the electron charge distribution is defined as

$$
\varrho(\boldsymbol{r})=\sum_{i=1}^{N} \Psi_{i}^{2}(\boldsymbol{r}) .
$$

Since the Laplace operator is linear, applying $\nabla^{2}$ to both sides of the above equation, we obtain the analogous relation to Eq. (9):

$$
\begin{gathered}
\nabla^{2} \varrho(\boldsymbol{r})=2 \sum_{i=1}^{N}\left\|\nabla \Psi_{i}(\boldsymbol{r})\right\|^{2} \\
+2 \sum_{i=1}^{N} \Psi_{i}(\boldsymbol{r}) \nabla^{2} \Psi_{i}(\boldsymbol{r}) .
\end{gathered}
$$

Integrating over the space $\mathbb{R}^{3}$ and applying Eq. (5) one obtains

$$
\begin{aligned}
& \sum_{i=1}^{N} \int_{\mathbb{R}^{3}} \Psi_{i}(\boldsymbol{r}) \nabla^{2} \Psi_{i}(\boldsymbol{r}) \mathrm{d} \boldsymbol{r} \\
& =-\sum_{i=1}^{N} \int_{\mathbb{R}^{3}}\left\|\nabla \Psi_{i}(\boldsymbol{r})\right\|^{2} \mathrm{~d} \boldsymbol{r} .
\end{aligned}
$$


Using Eq. (17) we get

$$
T=\frac{1}{2} \sum_{i=1}^{N} \int_{\mathbb{R}^{3}}\left\|\nabla \Psi_{i}(\boldsymbol{r})\right\|^{2} \mathrm{~d} \boldsymbol{r} .
$$

The above formula is more convenient than Eq. (17), since the degree of the differential operator is one (as compared to $\nabla^{2}$ in Eq. (17)). This reformulation is important when finite element method [14] or spectral method [15] are applied to solve the Kohn-Sham equation $[16]$.

\section{Summary}

We provided the short derivation of the von Weizsäcker formula. The derivation is based on the definition of the kinetic energy valid for any one-electron system. The derivation does not use the matrix density formulation. Moreover, it was shown that for finite system, containing any number of electrons, it holds $\int_{\mathbb{R}^{3}} \nabla^{2} \varrho(\boldsymbol{r}) \mathrm{d} \boldsymbol{r}=0$. Hence, the kinetic energy functional $T$ for any system does not need to account this term explicitly. Additionally, convenient formula for kinetic energy is provided which is particularly useful in finite element or spectral method.

\section{References}

[1] P. Hohenberg, W. Kohn, Phys. Rev. 136, B864 (1964).

[2] W. Kohn, L.J. Sham, Phys. Rev. 140, A1133 (1965).
[3] R.G. Parr, W. Yang, Density-Functional Theory of Atoms and Molecules, Oxford University Press, Oxford 1989 .

[4] W. Koch, M.C. Holthausen, A Chemist's Guide to Density Functional Theory, Wiley, New York 2000.

[5] Y.A. Wang, E.A. Carter, Progress in Theoretical Chemistry and Physics, Kluwer, Dordrecht 2000, p. 117.

[6] J. Nocedal, S.J. Wright, Numerical Optimization, Springer Series in Operations Research, Springer, New York 1999

[7] B. Zhou, Y.A. Wang, J. Chem. Phys. 128, 084101 (2008).

[8] B. Zhou, Y.A. Wang, J. Chem. Phys. 124, 081107 (2006).

[9] B. Zhou, Y.A. Wang, Int J. Quant. Chem. 107, 2995 (2007).

[10] H. Eschrig, The Fundamentals of Density Functional Theory, Teubner, Stuttgart 1996.

[11] N.H. March, Electron Density Theory of Atoms and Molecules, Academic, London 1992.

[12] G.M. Fichtenholz, Differential- und Integralrechnung, Harri, Berlin 1990.

[13] R.L. Liboff, Introductory Quantum Mechanics, Addison Wesley, New York 1987.

[14] I. Babuska, T. Strouboulis, The Finite Element Method and Its Reliability, Oxford University Press, Oxford 2001

[15] C. Canuto, M.Y. Hussaini, A. Quarteroni, T.A. Zang, Spectral Methods in Fluid Dynamics, Springer, New York 1988.

[16] Z. Romanowski, Modelling Simul. Mater. Sci. Eng. 16, 015003 (2008). 